\title{
Diffeomorphic Statistical Deformation Models
}

\section{Hansen, Michael Sass; Hansen, Mads/Fogtman; Larsen, Rasmus}

Published in:

2007 IEEE 11th International Conference on Computer Vision

Link to article, DOI:

10.1109/ICCV.2007.4409182

Publication date:

2007

Document Version

Publisher's PDF, also known as Version of record

Link back to DTU Orbit

Citation (APA):

Hansen, M. S., Hansen, MF., \& Larsen, R. (2007). Diffeomorphic Statistical Deformation Models. In 2007 IEEE 11th International Conference on Computer Vision (pp. 2626-2633). IEEE.

https://doi.org/10.1109/ICCV.2007.4409182

\section{General rights}

Copyright and moral rights for the publications made accessible in the public portal are retained by the authors and/or other copyright owners and it is a condition of accessing publications that users recognise and abide by the legal requirements associated with these rights.

- Users may download and print one copy of any publication from the public portal for the purpose of private study or research.

- You may not further distribute the material or use it for any profit-making activity or commercial gain

- You may freely distribute the URL identifying the publication in the public portal 


\title{
Diffeomorphic Statistical Deformation Models
}

\author{
Michael S. Hansen \\ Mads F. Hansen \\ Rasmus Larsen
Informatics and Mathematical Modelling, Technical University of Denmark Richard Petersens Plads, DTU - Bldg. 321, DK-2800 Kgs. Lyngby, DK \\ \{msh, mfh, rl\}@imm.dtu.dk
}

\begin{abstract}
In this paper we present a new method for constructing diffeomorphic statistical deformation models in arbitrary dimensional images with a nonlinear generative model and a linear parameter space.

Our deformation model is a modified version of the diffeomorphic model introduced by Cootes et al. The modifications ensure that no boundary restriction has to be enforced on the parameter space to prevent folds or tears in the deformation field.

For straightforward statistical analysis, principal component analysis and sparse methods, we assume that the parameters for a class of deformations lie on a linear manifold and that the distance between two deformations are given by the metric introduced by the $L_{2}$-norm in the parameter space. The chosen $L_{2}$-norm is shown to have a clear and intuitive interpretation on the usual nonlinear manifold.

Our model is validated on a set of MR images of corpus callosum with ground truth in form of manual expert annotations, and compared to Cootes's model.

We anticipate applications in unconstrained diffeomorphic synthesis of images, e.g. for tracking, segmentation, registration or classification purposes.
\end{abstract}

\section{Introduction}

Registration is the problem of establishing correspondence between points in different images. It has been used for building models of variation in groups of images for several years. Cootes et al. proposed the very successful active appearance models in 1998 [3], which, once trained, can establish correspondence between points in the model and the images using a piecewise affine mapping. Rueckert et al. presented a statistical deformation model based on registrations of an atlas to the images of the group [9]. Joshi et al. demonstrate how to construct an unbiased atlas from a population [5], and Cootes et al. presented a guaranteed diffeomorphic shape model [2] by using smooth kernels for interpolating a warp field and putting restrictions on the variation of the parameters. VesterChristensen et al. have presented an accelerated version of this algorithm [10], which is based on the inverse compositional method by Baker et al., which we have also made extensive use of in the presented work [1].

\section{Methods}

We define image registration as the identification of correspondence between positions in images. In the current work we address problems where the correspondences can be represented by a diffeomorphic function $f \in \mathcal{H}$, where $\mathcal{H}$ denotes the infinite dimensional group of diffeomorphisms on $\mathbb{R}^{N}$. The mapping from one image to the other is differentiable and the inverse exists and is also differentiable. Popular speaking this limits the problem of registration to the problem of finding smooth warps without folds or tears. More precisely this is fulfilled, when the Jacobean of the warp field is positive and well defined.

In the statistical analysis of the warp functions we are interested in estimating an unbiased atlas of the structures we are registering. We identify such an atlas as the groupwise maximizer of similarity between the atlas $R$ and the deformed images $I_{i}$, while minimizing the deformation fields $\phi_{i}$.

$$
\left[\phi_{i}, \hat{R}\right]=\min _{\phi_{i}, \hat{R}} \sum_{i} \mathcal{S}\left[\hat{R}, I_{i} \circ \phi_{i}\right]+\alpha \mathcal{D}\left(\phi_{i}\right)^{2} .
$$

where $\mathcal{S}$ denotes the similarity measure and $\mathcal{D}(\phi)$ denotes the regularization term, introduced to regularize the warp $\phi$ further than just restricting it to the space of the parameters, and $\alpha$ is the regularization parameter. 


\subsection{Parameterized diffeomorphisms}

Fletcher et al. have investigated geodesic curves on the nonlinear manifolds of the parameters of the M-reps parameterization [4]. Most of the current statistical analysis, however, is based on the assumption that the data is located on a linear manifold with the Euclidean metric, e.g. principal component analysis (PCA) and independent component analysis (ICA), which have nice properties as analytical tools. This is our motivation for introducing a function $\mathcal{G}$ which identifies $\mathbb{R}^{M}$ with a (hopefully interesting) subset of diffeomorphisms.

Let $\mathcal{H}\left(\mathbb{R}^{N}\right)$ denote the set of diffeomorphisms $(f$ : $\left.\mathbb{R}^{N} \rightarrow \mathbb{R}^{N}\right)$. Now let $\mathcal{G}$ be a bijective mapping:

$$
\mathcal{G}: \mathbb{R}^{M} \rightarrow \mathcal{H}_{t} .
$$

where $\mathcal{H}_{t}=\mathcal{G}\left(\mathbb{R}^{M}\right) \subset \mathcal{H}$. We let $\mathcal{H}_{t}$ inherit the Euclidean metric from the parameter space $\mathbb{R}^{M}$

$$
\begin{array}{r}
d\left(\mathcal{G}\left(t_{1}\right), \mathcal{G}\left(t_{2}\right)\right) \equiv d\left(t_{1}, t_{2}\right)=\left\|t_{1}-t_{2}\right\|_{2}, \\
t_{1}, t_{2} \in \mathbb{R}^{M} \text { and } \mathcal{G}\left(t_{1}\right), \mathcal{G}\left(t_{2}\right) \in \mathcal{H}_{t},
\end{array}
$$

from which we conclude that $\mathcal{G}$ is a homeomorphism, and that the spaces $\mathcal{H}_{t}=\mathcal{G}\left(\mathbb{R}^{M}\right)$ and $\mathbb{R}^{M}$ are topologically equivalent. To conclude it can be observed that the defined metric on the space of parameterized warps is the $L_{2}$ norm on $\mathbb{R}^{M}$ as intended.

\subsubsection{Composition of warps}

The composition of more diffeomorphisms is diffeomorphic, which is a very important property of diffeomorphisms in the present context.

$$
\begin{array}{r}
f_{i} \in \mathcal{H}, i \in\{1,2, \ldots, n\} \\
\phi=f_{n} \circ f_{n-1} \circ \ldots \circ f_{1} \Rightarrow \phi \in \mathcal{H}
\end{array}
$$

This allows for the construction of diffeomorphisms of higher complexity by the composition of several simpler warps. We shall assume we are dealing with parameterized warp functions, and our statistical analysis of warps can be reduced to the analysis of the warp parameters, in line with (3). For all images in our set the warp parameters shall warp from our reference, $R$, into the current target, $I$. In order to be able to compare parameters from different warp compositions it is evident that all our parameters exist in the same space. This is achieved by ensuring that all warps $f_{i}$ in a composition warp from the reference coordinate system[2].

\subsubsection{Grid based diffeomorphisms}

Several grid based representations of diffeomorphisms have been presented and they are commonly used at different levels of detail and composed succeedingly $[2,7,9]$. A general trait of the grid methods is that they manipulate the parameters of the functions describing the diffeomorphism, and that the functions have a local support in the image, either as points defined in the image or as basis functions with support around a control point. Often this parameterization of the grid is linear in the parameters and this obviously imposes some restrictions on the parameters to produce diffeomorphic warps. Cootes et al. specify a cut-off at displacements larger than $\frac{1}{\pi}$ of the cosine based kernel [2] and Lee et al. find a threshold bound on the B-spline parameters to secure that the B-spline based warp function is diffeomorphic [6].

\subsubsection{A proposed $\mathcal{G}$}

Let $\mathcal{F}$ be the function mapping from a real parameter space $\mathbb{R}^{M}$ into the space of functions from $\mathbb{R}^{N}$ to $\mathbb{R}^{N}$, e.g. in case of the B-spline warps, $\mathcal{F}$ maps from the parameter space into the space of $N$-dimensional Bspline functions $f: \mathbb{R}^{N}$ to $\mathbb{R}^{N}$, the image of $\mathcal{F}, \mathcal{K}$ can be shown to contain functions that are not diffeomorphic.

As discussed in the previous section there can for some parameterized warps be specified a threshold such that $\mathcal{P}=]-\tau_{1}, \tau_{1}[\times \cdots \times]-\tau_{M}, \tau_{M}\left[\right.$ and $\mathcal{F}: \mathcal{P} \rightarrow \mathcal{H}_{t}$, where $\mathcal{H}_{t} \equiv \mathcal{F}(\mathcal{P}) \subset \mathcal{H}$. In the current study we have investigated the use of a function $g: \mathbb{R}^{M} \rightarrow \mathcal{P}$, that is, a bounded monotonic injective function into the space of thresholded displacement parameters. Constructing $\mathcal{G}=\mathcal{F} \circ g$, where $\mathcal{G}: \mathbb{R}^{M} \rightarrow \mathcal{H}_{t}$ gives us the desired function $\mathcal{G}$, namely a homeomorphic mapping from the parameter space $\mathbb{R}^{M}$ into the space of diffeomorphisms. As an example of the function $g$ we have chosen a set of hyperbolic tangent function, because the range where it is close to linear is large. The composed mapping $\mathcal{G}$ and the different ranges are illustrated in Figure 1.

We define $g$ coordinate-wise by

$$
\begin{array}{r}
\left.\boldsymbol{g}=\left\{g_{1}, \ldots, g_{M}\right\} \text { where } g_{i}: R \rightarrow\right]-\tau_{i}, \tau_{i}[ \\
s_{i}=g_{i}\left(t_{i}\right)=\tau_{i} \tanh a_{i} t_{i}, \text { for } \quad i \in\{1, \ldots, M\}
\end{array}
$$

where $\tau_{i}$ are the threshold parameters reducing the displacement parameter space of the warp to $\mathcal{P} \subset \mathbb{R}^{M}$, $s=\left\{s_{1}, \ldots, s_{M}\right\} \in \mathcal{P}$ are the displacement parameters and $a_{i}$ are constants ensuring that the impact of each $t_{i}$ is of the same order of magnitude.

\subsubsection{Properties of the $g$ mapping}

Before we continue with an empirical validation of our proposed mapping we will make some theoretical considerations over the choice of homeomorphic mapping 


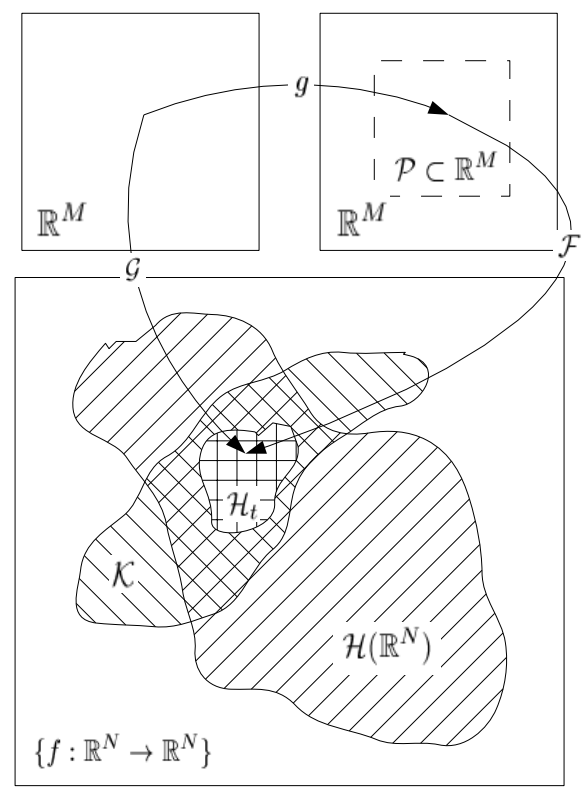

Figure 1. Illustration of the mapping $\mathcal{G}$ from $\mathbb{R}^{N}$ to $\mathcal{G}$, along with our proposed composed mapping $\mathcal{G}=\mathcal{F} \circ g$

$g$. For small values $t \in \mathbb{R}^{M}$ the $L_{2}$ norm in $\mathbb{R}^{M}$ is equivalent to a scaled $L_{2}$ norm in $g\left(\mathbb{R}^{M}\right)$ to a first order. In other words, relating this to diffeomorphic warps, for small deformations the defined norm is equivalent to the usual metric applied in analysis of the warp fields $[2,9]$.

\subsubsection{The parameter distribution}

We believe that the distribution of the parameters is well described by normal distribution, and we will show what distribution this describes in the displacement parameter space of the warp function. Let $f_{t_{i}}$ be the marginal distribution of the parameter $t_{i}$ and $f_{g_{i}}$ be the marginal distribution of the warp parameter $s_{i}=g_{i}\left(t_{i}\right)$, then

$$
\begin{array}{r}
f_{i}\left(t_{i}\right)=\frac{1}{\sqrt{2 \pi \sigma_{i}^{2}}} e^{-\frac{t_{i}^{2}}{2 \sigma_{i}^{2}}} \\
f_{g_{i}}\left(s_{i}\right)=\frac{1}{2 a_{i} \cdot \tau_{i} \sqrt{2 \pi \sigma_{i}^{2}}}\left(e^{-\frac{g^{-1}\left(s_{i}\right)^{2}}{2 \sigma_{i}^{2}}}+\right. \\
\left.\frac{e^{\frac{\mu_{i}^{2}}{2 \sigma_{i}^{2}}}}{2} e^{-\frac{\left(g^{-1}\left(s_{i}\right)-\mu_{i}\right)^{2}}{2 \sigma_{i}^{2}}}+\frac{e^{\frac{\mu_{i}^{2}}{2 \sigma_{i}^{2}}}}{2} e^{-\frac{\left(g^{-1}\left(s_{i}\right)+\mu_{i}\right)^{2}}{2 \sigma_{i}^{2}}}\right)
\end{array}
$$

where $\mu_{i}=\frac{\sigma_{i}^{2} a_{i}}{2}$ and this distribution is seen to be the composition of three Gaussian distributions scaled by $g^{-1}$. For small $\mu_{i}$ this is approaching the Gaussian distribution which is often the distribution for the warp parameters in the small deformation domain and for $\mu_{i}$ big the two $\mu_{i}$ displaced distributions dominate, and we observe a high concentration of parameters around the threshold $\tau_{i}$. In the presence of strong deformations this also what we expect when imposing a threshold on the warp deformation parameters. Based on these considerations we expect an $M$-dimensional normal distribution of our parameters to be well suited for modelling the distributions of the observed deformations.

\subsubsection{Statistical deformation model}

In the previous section we argued that the expected distribution of warps could be modelled as an $M$ dimensional normal distribution. If this is the case PCA is known to be the optimal choice of analysis tool for creating a compact model of the observations, and is therefore the method of choice in the current implementation.

\section{Implementation}

To validate our approach for construction of diffeomorphic deformation model we have adapted the grid based diffeomorphisms by Cootes [2] with our $g$ mapping. These diffeomorphisms can be viewed as an extension to standard linear interpolation, where the interpolation coefficients are transformed by a suitable kernel $k(r)$ which ensures smoothness across the grid boundaries. The displacement of a $2 \mathrm{D}$ point $\boldsymbol{x} \in \mathbb{R}^{2}$ is given by

$$
\begin{aligned}
\boldsymbol{u}(\boldsymbol{x}, \boldsymbol{d}) & =\sum_{m=0}^{1} \sum_{n=0}^{1} k_{n}(v) k_{m}(w) \boldsymbol{d}_{i+n, j+m} \\
& =\sum_{m=0}^{1} \sum_{n=0}^{1} a_{i+m, j+n}(\boldsymbol{x}) \boldsymbol{d}_{i+n, j+m} \\
& =\left[\begin{array}{cc}
\boldsymbol{a}(\boldsymbol{x})^{\top} & \mathbf{0} \\
\mathbf{0} & \boldsymbol{a}(\boldsymbol{x})^{\top}
\end{array}\right] \boldsymbol{d}
\end{aligned}
$$

where $k_{0}(r)=k(r), k_{1}(r)=1-k(r), i$ and $j$ is the local indices of the neighboring grid points, $v$ and $w$ are relative positions of $x$ in the neighborhood and $\boldsymbol{d}$ and $\boldsymbol{d}_{i, j}$ are all the displacements and the displacement of the $(i, j)$-node, respectively. By substituting the displacements $\boldsymbol{d}$ with the $g$ mapping with a suitable threshold $\tau$, this deformation model will no longer be able to generate non-diffeomorphisms. In the present example using the Cootes kernel, $\tau=1 / \pi$.

For notational simplicity the displacement in the $i$ th direction will represented by

$$
u_{i}\left(\boldsymbol{x}, \boldsymbol{t}_{i}\right)=\boldsymbol{a}(\boldsymbol{x})^{\top} \boldsymbol{g}_{\tau}\left(\boldsymbol{t}_{i}\right),
$$

and the warp function is written in the form

$$
\varphi(x, t)=x+u(x, t) .
$$




\subsection{Image registration}

To drive the registration between a reference image $R$ and a target image $I$ we apply the sum-of-squareddifferences (SSD) as our similarity measure and the regularization term is given by $\mathcal{D}(\phi)=d(e, \phi)=\|t\|_{2}$, where $e$ is the identity map corresponding to $\boldsymbol{t}=0$. The SSD comparison leads us to calculate the reference image as the arithmetic mean of the warped target images, as this is the optimum SSD solution to (1) [5].

$$
\begin{aligned}
F(\boldsymbol{t}) & =\frac{1}{2} \sum_{\boldsymbol{x}}\left(R(\boldsymbol{x})-I(\boldsymbol{\varphi}(\boldsymbol{x}, \boldsymbol{t}))^{2}+\alpha\|\boldsymbol{t}\|_{2}^{2}\right. \\
& =\frac{1}{2} \sum_{\boldsymbol{x}} E^{2}(\boldsymbol{x}, \boldsymbol{t})+\alpha\|\boldsymbol{t}\|_{2}^{2}
\end{aligned}
$$

To achieve a fast optimization we apply the inverse compositional optimization approach by Baker et al. [1] to the cost function. Thus, we obtain a minimum by iteratively minimizing

$$
\begin{aligned}
F_{i c}(\boldsymbol{t})= & \frac{1}{2} \sum_{\boldsymbol{x}}\left(R(\boldsymbol{\varphi}(\boldsymbol{x}, \Delta \boldsymbol{t}))-I(\boldsymbol{\varphi}(\boldsymbol{x}, \boldsymbol{t}))^{2}\right. \\
& +\alpha\left\|\boldsymbol{t}-\frac{\partial \boldsymbol{t}^{\prime}}{\partial \Delta \boldsymbol{t}} \Delta \boldsymbol{t}\right\|^{2}
\end{aligned}
$$

with respect to $\Delta \boldsymbol{t}$ and updating $\boldsymbol{t}$ according to

$$
\varphi\left(x, t^{\prime}\right) \leftarrow \varphi(x, t) \circ \varphi^{-1}(x, \Delta t) .
$$

In Appendix B it is shown how $\boldsymbol{t}^{\prime}$ is derived from (15).

By performing a first-order Taylor-expansion on $R(\boldsymbol{\varphi}(\boldsymbol{x}, \Delta \boldsymbol{t})$ around $\boldsymbol{x}$ in (14), taking the derivatives wrt. $\Delta t$ and setting them equal to zero we get

$$
\Delta \boldsymbol{t}=\boldsymbol{H}^{-} 1\left[\sum_{\boldsymbol{x}} \boldsymbol{S} \boldsymbol{D}(\boldsymbol{x})^{\top} E(\boldsymbol{x}, \boldsymbol{t})+\alpha{\frac{\partial \boldsymbol{t}^{\prime}}{\partial \Delta \boldsymbol{t}}}^{\top} \boldsymbol{t}\right]
$$

where

$$
\boldsymbol{S D}(\boldsymbol{x})=\nabla R(\boldsymbol{x}) \frac{\partial \varphi(\boldsymbol{x}, \mathbf{0})}{\partial \boldsymbol{t}}
$$

and

$$
\boldsymbol{H}=\sum_{\boldsymbol{x}} \boldsymbol{S} \boldsymbol{D}(\boldsymbol{x})^{\top} \boldsymbol{S} \boldsymbol{D}(\boldsymbol{x})+\alpha\left[\frac{\partial \boldsymbol{t}^{\prime}}{\partial \Delta \boldsymbol{t}}\right]^{\top}\left[\frac{\partial \boldsymbol{t}^{\prime}}{\partial \Delta \boldsymbol{t}}\right] .
$$

The advantages with this inverse compositional approach is that $\boldsymbol{S} \boldsymbol{D}(\boldsymbol{x})$ can be pre-computed as it is not dependent on $\boldsymbol{t}$.

\section{Validation: corpus callosum model}

To demonstrate our approach we have created a deformation model of the Corpus Callosum from 62 two dimensional MR images of the mid-sagittal crosssection of the corpus callosum brain structure. This data set is part of the LADIS (Leukoaraiosis and DISability) study [8], a pan-European study involving 12 hospitals and more than 700 patients. Furthermore, each corpus callosum have manually been annotated with 72 landmarks by a clinician, which we will later use for validation.

Prior to the non-rigid registration a rigid registration was performed to filter out non-anatomical variation. This was achieved by performing Procrustes analysis on the sets of annotation. After the rigid registration an initial reference was created by computing the mean image of the rigid registered images. All corpus callosum images were then non-rigidly registered to the reference, and a new reference was computed by averaging. This was done multiple times until the reference stabilized. For the non-rigid registration the cosine kernel $k(r)=0.5(1+\cos (\pi r))$ was applied [2]. The non-rigid warps were modelled by composing three grid based diffeomorphisms in a fine-to-coarse manner. The dimensions of the applied grids were $5 \times 4,10 \times 8$ and $20 \times 16$. The non-rigid registrations were carried out in coarse to fine order. After each level $\varphi_{i}$ of the warp was estimated the target image was updated by warping the target image back into the reference coordinate frame by $T_{n+1}(x)=T_{n}(\varphi(x))$. This was done to ensure that different parameters from different warps could be compared [2]. $a_{i}$ of the $g$ mapping was set proportional to the inverse of the squared grid node distance because the grid was 2 dimensional. The image registration was validated using the Dice measure, which is twice the intersecting area between the ground truth shape outline of the warped image and the outline of the reference shape divided by the total area inside the two outlines. The ground truth was obtained from the expert annotations. The Dice measure was $0.884 \pm 0.048$. In Fig. 2 we show an example of a typical registration of an image. In Fig. 3 the cumulative overlap of the aligned corpus callosum shapes before and after a rigid registration is illustrated, showing a clear improvement in correspondences between the shapes.

To create a compact deformation model, PCA was applied to the parameters after the groupwise registration of the images. 13 modes of variation could describe $95 \%$ of the observed variation in the population as observed in Figure 4, and the first three modes are illustrated in Fig. 5. The first mode of variation is seen to be related to a vertical stretch and in particular to the size of the septum pellucidum (the dark area between 


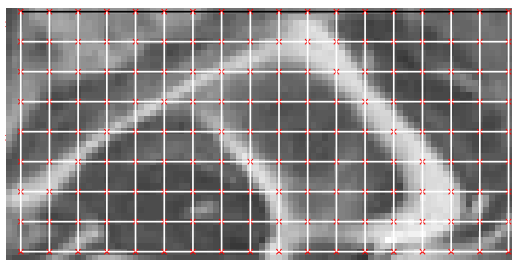

(a) Template image

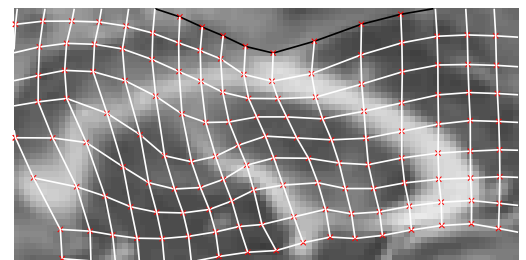

(b) Warped template

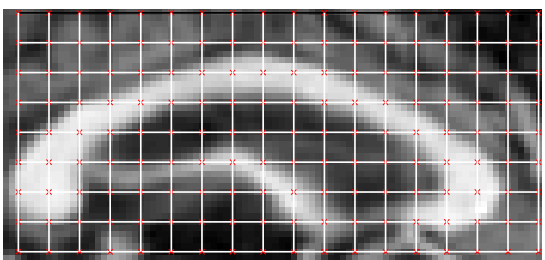

(c) Reference image

Figure 2. Registration of an image to the reference.

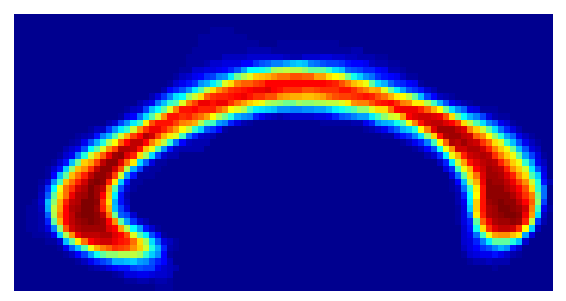

(a) Before nonrigid registration

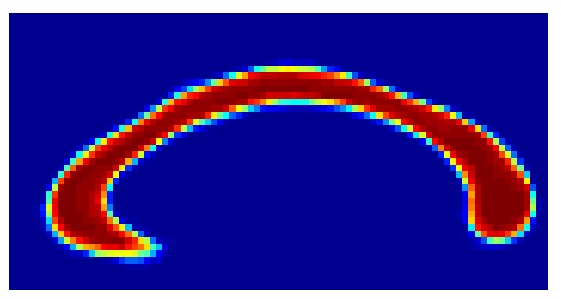

(b) After nonrigid registration

Figure 3. Cumulative overlap of the aligned corpus callosum shapes before and after a rigid registration

the bright corpus callosum and the bright Fornix), the second mode is related to the kink of the corpus callosum and the thickness of the structure and the same goes for the third mode but with a different bending of the Fornix. Rueckert et al. have also analyzed the corpus callosum and they found modes quite similar to the ones found in the current study [9]. For comparison we applied a regularized version of Cootes' algorithm to the same problem, and constructed a similar PCA model of the variation. The variance of the modes is nearly identical, as shown in Figure 4 and the obtained Dice scores were also the same. The major difference between the deformation modes are to be found where the warp displacement parameters are close to the limit $1 / \pi$. The sites in the Cootes warp with highest curvature are closer to singular than the same sites in the warp based on our parameterization.

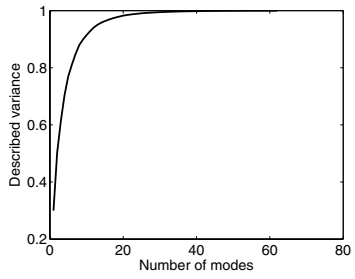

(a)

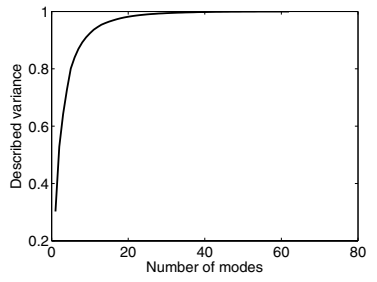

(b)
Figure 4. Plot relating described variance with number of modes included in the model. a: the presented method. b: method introduced by Cootes et al. [2].

\section{Discussion}

We have shown how a parametric function can be defined on the unbounded linear space $\mathbb{R}^{M}$ and still produce diffeomorphic warps. When this is accomplished by first mapping $\mathbb{R}^{M}$ into an open bounded subset of $\mathbb{R}^{M}$, which inevitably leads to an asymptotic behavior at the closure of the bounded set. In our implemented example the parameters of the model by Cootes et al. asymptotically approach $\frac{1}{\pi}$ where singularities in the warp may occur. We believe that our distance measure is very reasonable when we are indeed approaching a singularity, as a small change in the displacement parameters of the warp will cause a huge impact on curvature of the warp function. In Fig. 7, where -6 std. deviations of the first mode is shown. We see that a singularity start to form in the contracting area but this is highly unlikely as predicted by our model and metric.
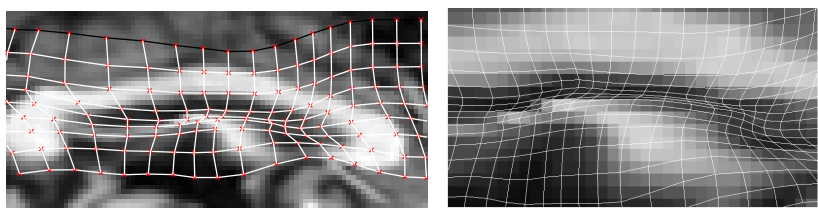

Figure 7. -6 Std. deviations of the first mode, normal view and a zoomed view on the beginning singularity.

With the choice of tanh function, the asymptotic behavior is assumed to be exponential, which may not always be the case. There are obviously an infinite variety of monotonic bounded functions, e.g. arcus tan- 


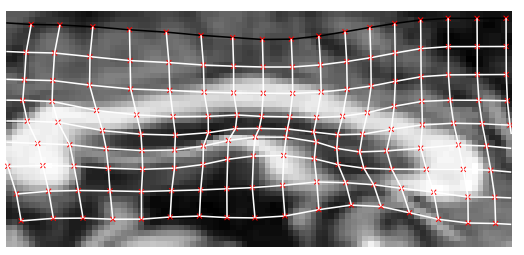

(a) 1st mode, -3 std. dev.

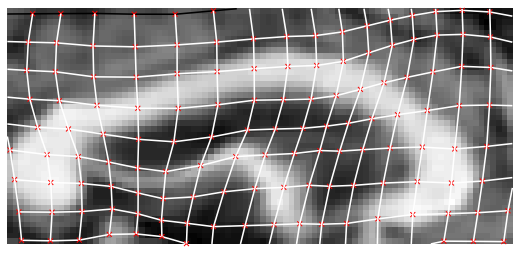

(b) 2nd mode, -3 std. dev.

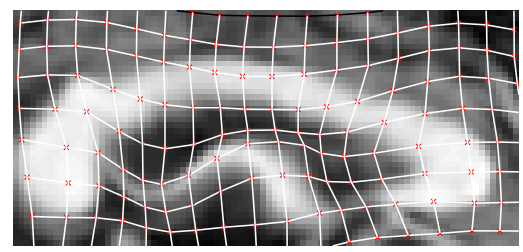

(c) 3rd mode, -3 std. dev.

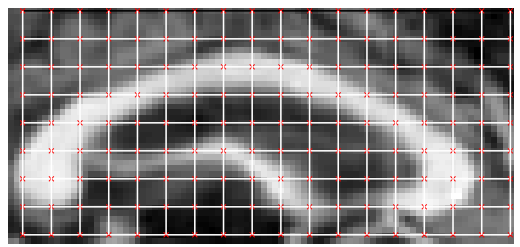

(d) Reference

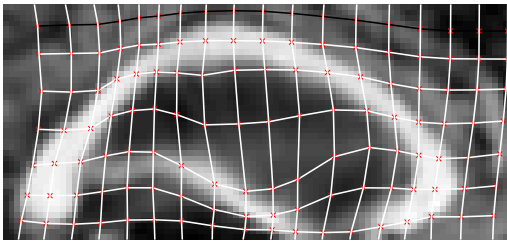

(e) 1 st mode, +3 std. dev.

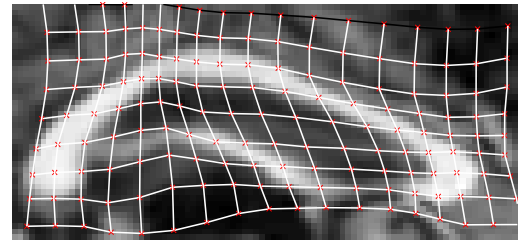

(f) 2nd mode, +3 std. dev.

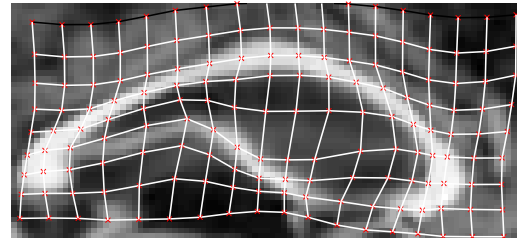

(g) 3rd mode, +3 std. dev.

Figure 5. First three modes of the corpus callosum deformation model estimated with the current method, shown as the reference warped \pm 3 std. deviations.

gent, and we will be investigating the choice of function in more detail.

A problem, we believe, that may occur with the proposed method is that we cannot be sure that the threshold does actually mark a singularity. A simple translation would for instance be asymptotic as well, which is why initial rigid alignment is very important indeed. Currently we investigate more involved parameter restrictions than the simple threshold to circumvent this possible problem.

Our validation on corpus callosum data showed that we were able to learn the important modes of variation, similar to previous obtained results, while the relatively high Dice coefficient illustrated that our warp representation was able to capture the large variations in the data set. We believe it is an advantage that all configurations in our parameter space are valid diffeomorphism, such that all gradients and derivatives during the optimization are well defined. Also we find it an advantage for tracking etc. that the the deformation as a function of the deformation model parameters is smooth, when using the presented method.

\section{Conclusions}

This paper proposed a new warp representation which allows statistical analysis on an unrestricted linear parameter space, where all derivatives are defined. Furthermore, we have shown that $L_{2}$-norm the parameter space introduces a reasonable metric in the actual space of modelled diffeomorphisms, and that our results compare well to those obtained using Cootes' deformation model.

\section{Acknowledgements}

The authors extend their gratitude to the LADIS work group for supplying the corpus callosum data. In particular we acknowledge the annotation effort of Charlotte Ryberg and Egill Rostrup from the Danish Research Center for Magnetic Resonance, Copenhagen University Hospital, Hvidovre, Denmark. We also wish to thank Vagn L. Hansen and Niels V. Christensen for fruitful discussion during this work.

\section{A. Warp inversion}

Theorem A.1. Consider the function $\varphi: \mathbb{R}^{N} \times \mathbb{R}^{M} \mapsto$ $\mathbb{R}^{N}$ of type $\boldsymbol{\varphi}(\boldsymbol{x}, \boldsymbol{t})=\boldsymbol{x}+\boldsymbol{u}(\boldsymbol{x}, \boldsymbol{t})$ and let $\varphi_{t}(\boldsymbol{x})=$ $\boldsymbol{\varphi}(\boldsymbol{x}, \boldsymbol{t})$ be a $\mathcal{C}^{1}$-diffeomorphism. If $\boldsymbol{u}(\boldsymbol{x}, \mathbf{0})=\mathbf{0}$ and 


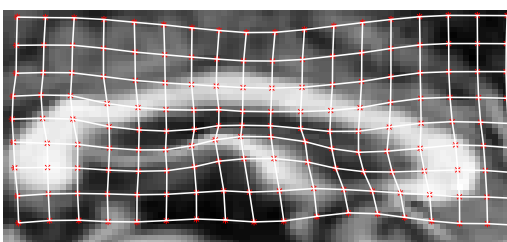

(a) 1 st mode, -3 std. dev

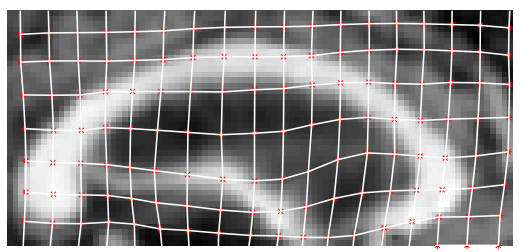

(b) 2nd mode, -3 std. dev.

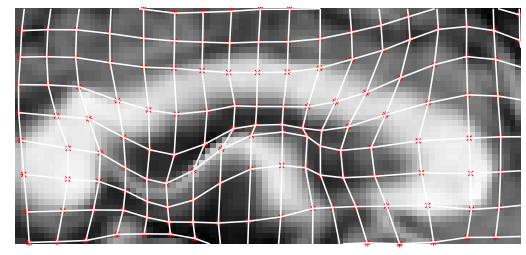

(c) 3rd mode, -3 std. dev.

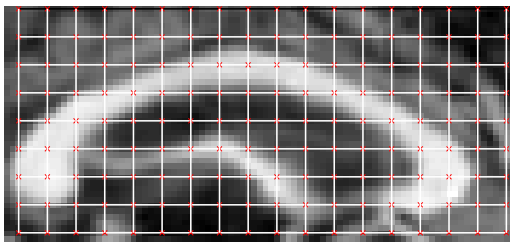

(d) Reference

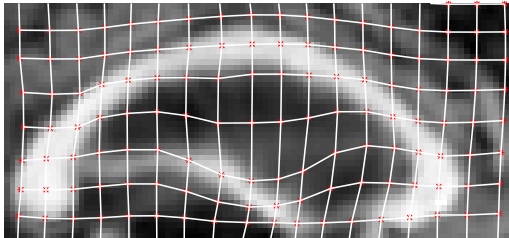

(e) 1 st mode, +3 std. dev.

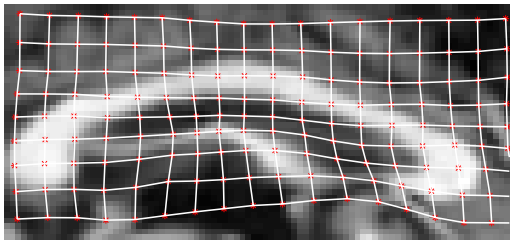

(f) 2nd mode, +3 std. dev.

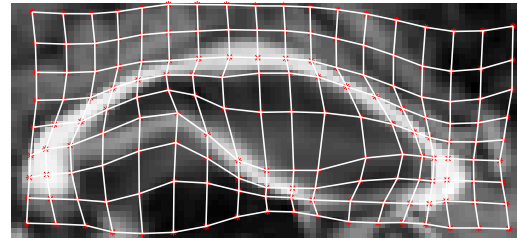

(g) 3rd mode, +3 std. dev.

Figure 6. First three modes of the corpus callosum deformation model estimated with a constrained version of Cootes' method, shown as the reference warped \pm 3 std. deviations

$\boldsymbol{u}(\boldsymbol{x}, \boldsymbol{t})=-\boldsymbol{u}(\boldsymbol{x}, \boldsymbol{t}), \boldsymbol{\varphi}(\boldsymbol{x},-\boldsymbol{t})$ converges with secondorder to $\boldsymbol{\varphi}^{-1}(\boldsymbol{x}, \boldsymbol{t})$.

Proof.

$$
\begin{aligned}
& \left|\xi_{i}(h \boldsymbol{t})\right|=\left|\varphi_{i}(\boldsymbol{\varphi}(\boldsymbol{x}, h \boldsymbol{t}),-h \boldsymbol{t})-x_{i}\right| \\
= & \left|x_{i}+u_{i}(\boldsymbol{x}, h \boldsymbol{t})-u_{i}(\boldsymbol{x}+\boldsymbol{u}(\boldsymbol{x}, h \boldsymbol{t}), h \boldsymbol{t})-x_{i}\right| \\
< & \left|u_{i}(\boldsymbol{x}, h \boldsymbol{t})-u_{i}(\boldsymbol{x}, h \boldsymbol{t})+\frac{\partial u_{i}}{\partial \boldsymbol{x}}(\boldsymbol{x}, h \boldsymbol{t}) \boldsymbol{u}(\boldsymbol{x}, h \boldsymbol{t})\right| \\
< & \left|h \boldsymbol{t}^{\top} \frac{\partial^{2} u_{i}}{\partial \boldsymbol{x} \partial h \boldsymbol{t}}(\boldsymbol{x}, \mathbf{0}) \frac{\partial \boldsymbol{u}}{\partial h \boldsymbol{t}}(\boldsymbol{x}, \mathbf{0}) h \boldsymbol{t}\right| \\
< & |c| \cdot\left|h^{2}\right|
\end{aligned}
$$

\section{B. Derivation of update function}

In general, it is unlikely that $\varphi(x, t) \circ \varphi^{-1}(\boldsymbol{x}, \Delta \boldsymbol{t})$ can be parameterized with $\boldsymbol{\varphi}\left(\boldsymbol{x}, \boldsymbol{t}^{\prime}\right)$, and thus it has to be approximated.

In Appendix A, it was shown that $\boldsymbol{\varphi}(\boldsymbol{x},-\boldsymbol{t})$ is a firstorder approximation to $\boldsymbol{\varphi}^{-1}(\boldsymbol{x}, \boldsymbol{t})$ as the error converges with second-order to zero. The composition in Eq. 15 is approximated with the parameters $\boldsymbol{t}^{\prime}$ which minimizes the SSD between the true composi- tional warp and the warp $\boldsymbol{\varphi}\left(\boldsymbol{x}, \boldsymbol{t}^{\prime}\right)$

$$
\sum_{x} \Delta \varphi(\boldsymbol{x})^{\top} \Delta \varphi(\boldsymbol{x})
$$

where

$$
\begin{aligned}
\Delta \varphi(\boldsymbol{x})= & \boldsymbol{\varphi}(\boldsymbol{\varphi}(\boldsymbol{x}, \Delta \boldsymbol{t}), \boldsymbol{t})-\boldsymbol{\varphi}\left(\boldsymbol{x}, \boldsymbol{t}^{\prime}\right) \\
= & \boldsymbol{a}(\boldsymbol{x})^{\top}\left(\boldsymbol{g}_{\tau}(\Delta \boldsymbol{t})-\boldsymbol{g}_{\tau}\left(\boldsymbol{t}^{\prime}\right)\right) \\
& +\boldsymbol{a}(\boldsymbol{\varphi}(\boldsymbol{x}, \Delta \boldsymbol{t})) \boldsymbol{g}_{\tau}(\boldsymbol{t})
\end{aligned}
$$

If

$$
\boldsymbol{A}=\left[\begin{array}{c}
\boldsymbol{a}\left(\boldsymbol{x}_{1}\right)^{\top} \\
\vdots \\
\boldsymbol{a}\left(\boldsymbol{x}_{\boldsymbol{n}}\right)^{\top}
\end{array}\right], \text { and } \boldsymbol{A}_{\varphi}=\left[\begin{array}{c}
\boldsymbol{a}\left(\boldsymbol{\varphi}\left(\boldsymbol{x}_{1}, \Delta \boldsymbol{t}\right)^{\top}\right. \\
\vdots \\
\boldsymbol{a}\left(\boldsymbol{\varphi}\left(\boldsymbol{x}_{n}, \Delta \boldsymbol{t}\right)^{\top}\right.
\end{array}\right]
$$

the updated warp parameters $\boldsymbol{t}^{\prime}$ can be found by solving the system

$$
\mathbf{0}=\boldsymbol{A}\left(\boldsymbol{g}_{\tau}\left(\Delta \boldsymbol{t}_{i}\right)-\boldsymbol{g}_{\tau}\left(\boldsymbol{t}_{i}^{\prime}\right)\right)+\boldsymbol{A}_{\varphi} \boldsymbol{g}_{\tau}\left(\boldsymbol{t}_{i}\right)
$$

The least square solution to the system is

$$
\boldsymbol{t}_{i}^{\prime}=\boldsymbol{g}_{\tau}^{-1}\left(\boldsymbol{A}^{\dagger} \boldsymbol{A}_{\varphi} \boldsymbol{g}_{\tau}\left(\boldsymbol{t}_{i}\right)+\boldsymbol{g}_{\tau}\left(\Delta \boldsymbol{t}_{i}\right)\right)
$$

where $\boldsymbol{A}^{\dagger}=\left[\boldsymbol{A}^{\top} \boldsymbol{A}\right]^{-1} \boldsymbol{A}^{\top}$. 
As $A_{\varphi}$ has to be evaluated on warped points it is relatively computational expensive to evaluate. Thus, we perform a first-order Taylor expansion on $A_{\varphi}$ and arrive at

$$
\boldsymbol{t}_{i}^{\prime}=\boldsymbol{k}^{-1}\left(\boldsymbol{A}^{\dagger} \boldsymbol{A}_{J_{i}} \boldsymbol{A} \boldsymbol{g}_{\tau}\left(\Delta \boldsymbol{t}_{i}\right)+\boldsymbol{g}_{\tau}\left(\Delta \boldsymbol{t}_{i}\right)+\boldsymbol{g}_{\tau}\left(\boldsymbol{t}_{i}\right)\right),
$$

where

$$
A_{J_{i}}=\boldsymbol{I}+\operatorname{diag}\left(\frac{\partial \boldsymbol{a}\left(\boldsymbol{x}_{j}\right)^{\top}}{\partial x_{i}} \boldsymbol{g}_{\tau}\left(\boldsymbol{t}_{i}\right)\right)_{j=1 \ldots n}
$$

\section{References}

[1] S. Baker and I. Matthews. Lucas-kanade 20 years on A unifying framework. International Journal of Computer Vision, 56(3):221-255, 2004. 1, 4

[2] T. Cootes, C. Twinning, and C. Taylor. Diffeomorphic statistical shape models. British Machine Vision Conference, 1:447-456, 2004. 1, 2, 3, 4, 5

[3] T. F. Cootes, G. J. Edwards, and C. J. Taylor. Active appearance models. 5th European Conference on Computer Vision, 2:484-498, 1998. 1

[4] P. T. Fletcher, C. Lu, S. M. Pizer, and S. Joshi. Prinicpal geodesic analysis for the study of nonlinear statistics of shape. IEEE Transactions on Medical Image Analysis, 23(8):995-1005, 2004. 2

[5] S. Joshi, B. Davis, M. Jomier, and G. Gerig. Unbiased diffeomorphic atlas construction for computational anatomy. Neuroimage, 23:151-160, 2004. 1,4

[6] S. Lee, G. Wolberg, K.-Y. Chwa, and S. Y. Shin. Image metamorphosis with scattered feature constraints. IEEE Trans. Visualizat. Comput. Graphics, 2(4):337 Ü354, 1996. 2

[7] J. Modersitzki. Numerical methods for image registration. Oxford Uni. Press, 2004. 2

[8] L. Pantoni, A. M. Basile, G. Pracucci, K. Asplund, J. Bogousslavsky, H. Chabriat, T. Erkinjuntti, F. Fazekas, J. M. Ferro, M. Hennerici, J. O'brien, P. Scheltens, M. C. Visser, L. O. Wahlund, G. Waldemar, A. Wallin, and D. Inzitari. Impact of age-related cerebral white matter changes on the transition to disability - the LADIS study: Rationale, design and methodology. Neuroepidemiology, 24(1-2):51-62, 2005. 4

[9] D. Rueckert, A. F. Frangi, and J. A. Schnabel. Automatic construction of $3 \mathrm{D}$ statistical deformation models of the brain using nonrigid registration. IEEE Transactions on Medical Imaging, 22(8):1014-25, 2003. 1, 2, 3, 5
[10] M. Vester-Christensen, S. G. Erbou, S. Darkner, and R. Larsen. Accelerated 3D image registration. In International Symposium on Medical Imaging 2007, San Diego, $\{C A\}$, feb 2007. 1 